\title{
Multiwavelength Detection of Cluster AGN
}

\author{
Alison J. Klesman ${ }^{1}$ and Vicki L. Sarajedini ${ }^{1}$ \\ ${ }^{1}$ Department of Astronomy, University of Florida, 211 Bryant Space Science Center, \\ Gainesville, FL 32611, USA \\ Email: alichan, vicki@astro.ufl.edu
}

\begin{abstract}
We present the initial results of a multiwavelength study to detect AGN in several galaxy clusters at moderate redshifts $(0.5<z<0.9)$. By using multiple epochs of $H S T$ data, we identify $10-15$ optically variable galactic nuclei in each cluster. The variable and non-variable galaxies are compared with X-ray point sources.
\end{abstract}

Keywords. galaxies: active, galaxies: clusters: general

The fraction of AGN in rich clusters has implications for determining the primary fueling mechanism for AGN (Giovanelli \& Haynes 1985). Fueling arguments suggest that clusters should contain fewer AGN than the field. Among the clusters in this study, we find that up to $\sim 3 \%$ of all cluster members exhibit significant optical variability and $2-7 \%$ of objects with optical counterparts are detected as X-ray point sources. This number is a lower limit, as many of the optical variables do not currently have spectroscopic information to determine cluster membership. These numbers are comparable to optical variability studies in the field (Sarajedini et al. 2006) and recent cluster X-ray studies (e.g., Martini et al. 2006). Averaged over five clusters (Figure 1), it can be seen that the fraction of enclosed AGN rises more quickly with radius than for galaxies that do not exhibit AGN behavior. A more complete census of AGN in clusters, including identification of obscured sources, is necessary to quantify the fraction of galaxies with sufficient fueling material and place limits on the efficiency of gas stripping in clusters.
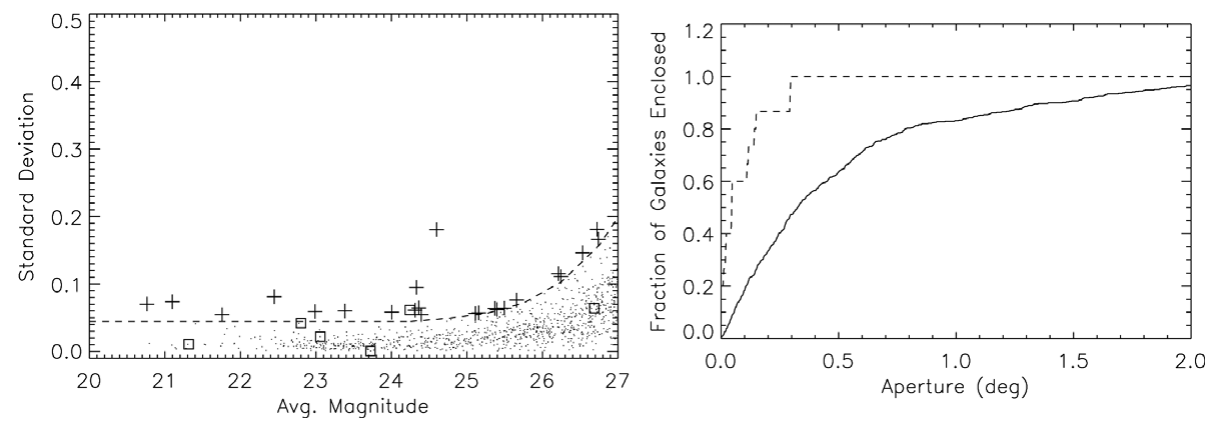

Figure 1. Left: Standard deviation vs. average magnitude in MACSJ0717+3745. Crosses are optical variables without X-ray counterparts; squares are X-ray sources with optical counterparts. The dashed line is the variability threshold. Right: Enclosed fraction of confirmed cluster members averaged over 5 clusters based on $10^{\prime \prime}$ circular apertures centered around the BCG. The solid histogram shows non-AGN (529); the dashed histogram shows AGN candidates (14).

\section{References}

Giovanelli, R. \& Haynes, M. P. 1985, ApJ, 292, 404

Martini, P., et al. 2006, ApJ, 644, 116

Sarajedini, V. L., et al. 2006, ApJS, 166, 69 